\title{
Racial/ethnic differences in health insurance adequacy and consistency among children: Evidence from the 2011/12 National Survey of Children's Health
}

\author{
Tulay G. Soylu, Eman Elashkar, Fatemah Aloudah, Munir Ahmed, Panagiota Kitsantas \\ Department of Health Administration and Policy, George Mason University, Fairfax VA, USA
}

Significance for public health

As the number of minority US children increases, monitoring racial/ethnic differences in health insurance coverage becomes critical in creating insurance programs that can provide adequate and consistent coverage. Using a nationally representative sample, the findings of this study suggest that low income and poor maternal health can adversely affect insurance consistency and adequacy for both minority and white children. This indicates that research studies on inequalities of healthcare coverage should also focus on underserved white populations of children as their insurance coverage is affected by similar factors as those for minority children. Elimination of inequalities may require targeted interventions that include the well-being of the entire family, cross-cultural education of healthcare providers, policy changes to grant low-income children with appropriate and reliable health insurance, and an ongoing monitoring of disparities by health plans.

\footnotetext{
Abstract

Background: Surveillance of disparities in healthcare insurance, services and quality of care among children are critical for properly serving the medical/healthcare needs of underserved populations. The purpose of this study was to assess racial/ethnic differences in children's ( 0 to 17 years old) health insurance adequacy and consistency (child has insurance coverage for the last 12 months)

Design and methods: We used data from the 2011/2012 National Survey of Children's Health $(n=79,474)$. Descriptive statistics and logistic regression analyses were conducted to examine the distribution and influence of several sociodemographic/family related factors on insurance adequacy and consistency across different racial/ethnic groups.

Results: Stratified analyses by race/ethnicity revealed that white and black children living in households at or below $299 \%$ of the Federal Poverty Level (FPL) were approximately 29 to $42 \%$ less likely to have adequate insurance compared to children living in families of higher income levels. Regardless of race/ethnicity, we found that children with public health insurance were more likely to have adequate insurance than their privately insured counterparts, while adolescents were at greater risk of inadequate coverage. Hispanic and black children were more likely to lack consistent insurance coverage.

Conclusions: This study provides evidence that racial/ethnic differences in adequate and consistent health insurance exists with both white and minority children being affected adversely by poverty. Establishing outreach programs for low income families, and cross-cultural education for healthcare providers may help increase health insurance adequacy and consistency within certain underserved populations.
}

\section{Introduction}

Recent estimates indicate that 3.3 million children under 18 years old are uninsured in the U.S. ${ }^{1}$ The lack of health insurance has deleterious effects on children's health and well-being. ${ }^{2,3}$ Children who lack health insurance coverage are more likely to experience adverse health outcomes and have limited access to preventive services compared to those who are insured. ${ }^{3}$ Children without consistent health insurance (e.g., those with periods of no insurance) also have a greater likelihood for unmet health care needs and receipt of preventive services. ${ }^{4-6}$

Minority children, specifically, tend to have higher rates of suboptimal dental and medical health as well as no usual source of care when compared to other racial/ethnic groups of children. ${ }^{7}$ As it is evidenced by a recent U.S. Census Bureau report, 53\% of uninsured children are Hispanic and black. ${ }^{8}$ Among those insured, Hispanic and older children are more likely to be underinsured. ${ }^{9}$ Recent studies indicate that black and Hispanic children have a lower likelihood of not receiving all needed medical/dental care and specialty care compared to white children. ${ }^{10}$

Efforts to reduce disparities in access to quality healthcare are reflected with the implementation of the Affordable Care Act (ACA) which is designed to promote access to equitable and more efficient health care. The ACA invested in initiatives that aim to increase the racial and ethnic diversity of health care providers and provide them with strengthened cultural competency training. ${ }^{11}$ However, despite these efforts, research studies show a wide gap in health care access, quality, and outcomes. ${ }^{12}$

Limited research exists on factors that may influence underinsurance and inconsistencies in healthcare coverage, and overall access to quality healthcare among children. Although most racial/ethnic minority children are eligible for Medicaid and Children's Health Insurance Program (CHIP), many are not aware of their eligibility, while others may face barriers in enrolling in such programs due to limited English proficiency. ${ }^{13}$ The present study was designed to address this gap in research and provide population-based evidence for policy makers and potential interventions targeting children who are at risk for not having adequate or consistent health insurance.

The purpose of the present study was to examine racial/ethnic differences in adequacy and consistency of public or private health insurance, and assess associations of several demographic and family related factors with insurance adequacy and consistency across different racial/ethnic groups. We used data from the 2011/2012 National Survey of Children's Health (NSCH). ${ }^{14}$ 


\section{Materials and Methods}

The 2011/2012 NSCH is a cross-sectional telephone survey of US households with at least one resident child aged 0 to 17 years old at the time of the interview. The goal of this survey is to assess the physical and emotional health of children, as well as factors that may relate to child well-being, including medical homes, family interactions, parental health, school and after-school experiences, and neighborhood characteristics. The NSCH uses the same sampling frame as the Centers for Disease Control and Prevention (CDC) National Immunization Survey (NIS). Using a complex survey design, the 2011/2012 NSCH surveyed the parents of a total of 95,677 children, or approximately 2000 per state. Details on the sampling design and procedures of this survey are presented at the 2011/2012 NSCH Guide (NSCH, 2011-2012). ${ }^{15}$

The analytic sample of this study $(n=79,474)$ excluded uninsured children and missing data on variables for which it ranged from $0.05 \%$ to $9 \%$ There were 3464 uninsured children who were excluded from this analytic sample since this study aimed to analyze the adequacy and consistency of health insurance coverage. This sample selection approach is in line with previous research in the field. ${ }^{9}$ Further, multicultural racial/ethnic groups $(n=10,446)$ were excluded from the analytical sample since there were several groups which were consisted of small sample sizes. Responses of don't know and refuse to answer were considered to be missing data $(n=2293)$.

\section{Measures}

The outcome variables in this study included adequacy of health insurance and consistency of health insurance. We included multiple dimensions in the definition of insurance adequacy and consistency as cited by the American Academy of Pediatrics and previous studies. ${ }^{9}, 16$

The outcome variables of adequacy and consistency were created and provided for analysis in this study by the NSCH research team (NSCH, 2011-2012). Appendix A provides a summary of these outcome variables. The adequacy variable was created from parents' or guardians' responses to four questions: Does the child's health insurance offer benefits or cover services that meet his or her needs? Does the child's health insurance allow him or her to see the health care providers he or she needs? Not including health insurance premiums or costs that are covered by insurance, do you pay any money for the child's health care? A positive answer to the third question led to a follow-up question, How often are these costs reasonable? If a parent or guardian indicated that the out-ofpocket costs were always or usually, reasonable and responded as always or usually to the other questions listed above, then the child was considered to have adequate insurance coverage. Other research studies have used the same approach in measuring adequacy. 9,17 The combination of these survey questions led to an outcome variable that consisted of two categories: adequate (current insurance is adequate for child's needs, $n=61,268$ ), and not adequate or underinsured $(\mathrm{n}=17,913)$. The outcome variable adequacy did not include uninsured children, refused to answer and missing records. The second outcome variable, consistency of health insurance, measured whether a child had consistent health insurance coverage during the past 12 months (NSCH, 2011-2012, www.childhealthdata.org). It was created by the NSCH team using the following survey questions: Does the child have any kind of health care coverage, including health insurance, prepaid plans such as HMOs, or government plans such as Medicaid? which consisted of three parts: 1) If YES, Is that coverage for him/her insured by Medicaid or the Children's Health Insurance Program, CHIP?, 2) If YES, During the past 12 months, was there any time when he/she was not covered by any health insurance?, 3) If NO, During the past 12 months, was there any time when he/she had health care coverage? The combinations of these survey questions resulted in the dichotomous variable (yes/no) for consistency of health insurance in the past 12 months: consistent (child had consistent health insurance coverage during the past 12 months, $\mathrm{n}=75,509)$, and not consistent $(\mathrm{n}=3541)(\mathrm{NSCH}, 2011-2012)$.

The independent variables considered in this study were: race/ethnicity with three groups: white, black, Hispanic children; children's age classified as 0-5, 6-11, and 12-17 years old; gender (male and female); type of health insurance included public insurance and private insurance; poverty level which was constructed based on the family's income and included 0-199\% Federal Poverty Level (FPL), 200-299\% FPL, 300-399\% FPL, 400\% FPL; family structure consisted of two parent - biological, two parent step family, single mother - no father, and other family type; and maternal health status (both mental and physical status). These independent variables were selected based on previous research in the field. ${ }^{18-22}$

\section{Data analysis}

Descriptive statistics and Chi-square analysis were conducted to describe the sample and test for significant associations between the two main outcome variables and sample characteristics. Stratified analysis was performed based on children's racial/ethnic background to examine associations between adequacy and consistency of health insurance with children's characteristics. Several logistic regression models were built separately for each racial/ethnic group to determine how children's characteristics and those of their families influence their healthcare insurance adequacy and consistency status. All analyses were weighted to account for the complex survey design of the 2011-2012 NSCH. The STATA software was used to analyze the data. ${ }^{23}$

\section{Results}

Among the children who were insured (94.5\%), 57.4\% of them were privately insured whereas $37.1 \%$ were publicly insured. The uninsured children $(3.6 \%)$ were not part of this study, and it was excluded from the analytic sample. Overall, most of the parents reported that their children's insurance was adequate $(77.4 \%)$ and consistent $(95.5 \%)$ during the past year. Racial/ethnic differences in sociodemographic/family characteristics are presented in Table 1. A significantly larger percent of Hispanic (39.9\%) and black (23.2\%) children had public insurance, while most white children were privately insured (74.9\%). More than half of the Hispanic and black children lived in households at or below the 299\% FPL and this was significantly higher than their white counterparts. White and Hispanic children had also some of the highest rates of living with mothers not being at good/excellent mental and physical health.

\section{Adequacy of health insurance}

Table 2 displays the distribution of children's characteristics, family structure, type of health insurance and their mother's health status by adequacy of healthcare insurance. A significantly larger percent of $12-17$ year-old children $(26.4 \%)$ as well as males (23.8\%), children of Hispanic origin $(24.3 \%)$ and those with private insurance $(26.8 \%)$ lacked adequate health insurance coverage. Similarly, a large percent of children living in families between 200-399\% of the FPL (approximately 53.2\%) and those with mothers not having good/excellent physical and mental health $(25.8 \%)$ 
did not have adequate health insurance in the past year. The association between race/ethnicity and insurance adequacy was not statistically significant.

Stratified logistic regression analyses by race/ethnicity (Table 3) revealed that black female children were 1.36 (95\% Confidence Interval (CI): $1.12,1.67)$ times more likely to have adequate insurance than their male counterparts. No significant associations between gender and insurance adequacy were found for the other groups. Further, black children living in a household with a single mother were $1.43(95 \% \mathrm{CI}: 1.13,1.81)$ times more likely to have insurance adequacy relative to those residing in a two-parent household (biological parents). White and black children living in households at or below $299 \%$ of the FPL were approximately 29 to $42 \%$ less likely to have adequate insurance compared to children living in families at or above $400 \%$ FPL. Poverty level and insurance adequacy were not significantly associated within the Hispanic group. Furthermore, Hispanic and white children living with mothers of poor mental and physical health were less likely to have adequate health insurance compared to children whose mothers' health status was good/excellent. All children (regardless of race/ethnicity) between the ages of 12-17 years old had a decreased likelihood of having adequate health insurance compared to younger children, whereas children with public insurance were at least twice as likely to have insurance adequacy compared to children with private insurance.

\section{Consistency of health insurance}

Associations between children's characteristics and consistency of health insurance revealed that Hispanic (9.1\%) and black (7.9\%) children, those with public insurance (11.3\%) and children living in households at or below the $299 \%$ FPL were significantly less likely to have consistent insurance in the past 12 months (Table 4). A significantly higher percent of children living in families other than two parent-biological (percent ranged from 6.7 to $8.5 \%$ ) had gaps in their insurance. Further, children whose mothers were not in good/excellent mental and physical health were less likely to report consistent healthcare insurance.

Table 5 presents findings related to frequency distribution and logistic regression analysis for health insurance consistency. Regardless of racial/ethnic background, children with public insurance were less likely to report consistency of health insurance compared to those with private insurance. Higher poverty levels for white and Hispanic children significantly decreased their likelihood for consistent health insurance status compared to those living at $400 \%$ FPL and above. Hispanic children whose mothers had poor mental and physical health were 0.64 (95\% CI: $0.45,0.91)$ times less likely to have consistent health insurance in comparison to those whose mothers were in good health. No significant associations were observed between age, gender and family structure with health insurance consistency for any of the racial/ethnic groups in the logistic regression models.

\section{Discussion}

This study assessed associations between children's sociodemographic and family characteristics with adequacy and consistency of health insurance coverage within the context of race/ethnicity. It builds on previous research on this subject and adds to the understanding of the role of these characteristics within the context of race/ethnicity in influencing healthcare insurance coverage. ${ }^{9,18,19}$

We found that adolescents across all racial/ethnic groups were less likely to have adequate insurance compared to younger children. This has been observed in previous studies and it may be an indicator of needed services that are not covered normally by insurance (e.g., mental health care). ${ }^{9}$ Although most states have introduced some form of CHIP to cover children less than five years of age, it may not be sufficient for older children. This highlights the need to focus on older children to ensure adequate

Table 1. Racial/ethnic associations with socio-demographic/family characteristics.

\begin{tabular}{|c|c|c|c|c|}
\hline & White & $\begin{array}{l}\text { d Count (W } \\
\text { Hispanic }\end{array}$ & Black & P-value \\
\hline $\begin{array}{l}\text { Age } \\
0-5 \text { years old } \\
6-11 \text { years old } \\
12-17 \text { years old }\end{array}$ & $\begin{array}{l}17,704(57.0) \\
18,825(58.5) \\
22,853(63.0)\end{array}$ & $\begin{array}{l}4,360(29.1) \\
3,887(25.9) \\
3,336(20.9)\end{array}$ & $\begin{array}{l}2,610(13.9) \\
2,860(15.6) \\
3,039(16.1)\end{array}$ & 0.0000 \\
\hline $\begin{array}{l}\text { Gender } \\
\text { Male } \\
\text { Female } \\
\end{array}$ & $\begin{array}{l}30,612(60.0) \\
28,705(59.1)\end{array}$ & $\begin{array}{l}6,035(25.3) \\
5,542(25.1)\end{array}$ & $\begin{array}{l}4,371(14.7) \\
4,135(15.8)\end{array}$ & 0.2457 \\
\hline $\begin{array}{l}\text { Type of healthcare insurance } \\
\text { Public insurance } \\
\text { Private Insurance }\end{array}$ & $\begin{array}{l}12,437(36.9) \\
46,452(74.9)\end{array}$ & $\begin{array}{l}6,149(39.9) \\
5,187(15.2)\end{array}$ & $\begin{array}{c}4,618(23.2) \\
3,739(9.9)\end{array}$ & 0.0000 \\
\hline $\begin{array}{l}\text { Poverty level } \\
\text { 0-199\% FPL } \\
200-299 \% \text { FPL } \\
300-399 \% \text { FPL } \\
\text { 400\% FPL and above }\end{array}$ & $\begin{array}{l}14,145(39.8) \\
10,056(66.3) \\
9,750(74.0) \\
25,431(79.6)\end{array}$ & $\begin{array}{l}6,606(39.0) \\
1,510(19.8) \\
1,066(14.2) \\
2,401(12.2)\end{array}$ & $\begin{array}{l}4,489(21.2) \\
1,258(13.9) \\
880(11.8) \\
1,882(8.2)\end{array}$ & 0.0000 \\
\hline $\begin{array}{l}\text { Family Structure } \\
\text { Two parents - biological } \\
\text { Two parents - step family } \\
\text { Single mother - no father } \\
\text { Other family type }\end{array}$ & $\begin{array}{l}45,243(66.3) \\
3,931(61.6) \\
6,585(38.4) \\
3,380(53.0)\end{array}$ & $\begin{array}{c}7,477(25.2) \\
824(22.1) \\
2,461(27.9) \\
742(20.6)\end{array}$ & $\begin{array}{c}3,269(8.5) \\
684(16.3) \\
3,389(33.7) \\
1,107(26.4)\end{array}$ & 0.0000 \\
\hline $\begin{array}{l}\text { Maternal health status (mental and physical) } \\
\text { BOTH Good/Excellent } \\
\text { BOTH Not Good/Excellent }\end{array}$ & $\begin{array}{l}37,187(67.8) \\
18,828(49.6)\end{array}$ & $\begin{array}{l}5,143(19.4) \\
5,682(33.9)\end{array}$ & $\begin{array}{l}3,831(12.8) \\
3,559(16.5)\end{array}$ & 0.0000 \\
\hline
\end{tabular}


Table 2. Distribution of children's sociodemographic/family characteristics by health insurance adequacy in 2011/12 NSCH.

\begin{tabular}{|c|c|c|c|}
\hline & Adequ & surance; Unwei & $\%)$ \\
\hline & Adequate & Not Adequate & P-value \\
\hline Age & & & 0.0000 \\
\hline $0-5$ years old & $20,200(81.1)$ & 4,391 (18.9) & \\
\hline 6 -11 years old & $19,387(76.1)$ & $6,096(23.9)$ & \\
\hline $12-17$ years old & $21,681(73.6)$ & $7,426(26.4)$ & \\
\hline Gender & & & 0.0381 \\
\hline Male & $31,520(76.2)$ & $9,348(23.8)$ & \\
\hline Female & $29,695(77.6)$ & $8,544(22.4)$ & \\
\hline Race/ethnicity & & & 0.1283 \\
\hline White & $45,853(77.1)$ & $13,367(22.9)$ & \\
\hline Hispanic & $8,835(75.8)$ & $2,662(24.2)$ & \\
\hline Black & $6,580(78.1)$ & $1,884(21.9)$ & \\
\hline Type of healthcare insurance & & & 0.0000 \\
\hline Public insurance & $19,577(82.5)$ & $3,605(17.5)$ & \\
\hline Private insurance & $41,173(73.2)$ & $14,173(26.8)$ & \\
\hline Poverty Level & & & 0.0000 \\
\hline $0-199 \%$ FPL & $19,981(78.7)$ & $5,095(21.3)$ & \\
\hline $200-299 \%$ FPL & $9,339(74.1)$ & $3,417(25.9)$ & \\
\hline 300-399\% FPL & $8,604(72.7)$ & $3,054(27.3)$ & \\
\hline 400\% FPL and above & $23,344(77.7)$ & $6,347(22.3)$ & \\
\hline Family structure & & & 0.0110 \\
\hline Two parents - biological & $42,909(76.0)$ & $12,907(24.0)$ & \\
\hline Two parents - step family & $4,165(78.0)$ & $1,254(22.0)$ & \\
\hline Single mother - no father & $9,726(78.8)$ & $2,655(21.2)$ & \\
\hline Other family type & $4,177(78.4)$ & $1,009(21.6)$ & \\
\hline Maternal health status (mental and physical) & & & 0.0000 \\
\hline BOTH Good/Excellent & $36,445(78.7)$ & $9,581(21.3)$ & \\
\hline BOTH Not Good/Excellent & $20,674(74.2)$ & $7,285(25.8)$ & \\
\hline
\end{tabular}

Table 3. Weighted analysis of health insurance adequacy and odds ratios (OR) (95\% Confidence Intervals) by race/ethnicity among children in 2011/12 NSCH.

\begin{tabular}{|c|c|c|c|c|}
\hline & $\begin{array}{l}\text { Health Insurance } \\
\text { Adequacy, White }\end{array}$ & \multicolumn{2}{|c|}{$\begin{array}{l}\text { Health Insurance } \\
\text { Adequacy, Hispanic }\end{array}$} & \multirow{2}{*}{$\begin{array}{l}\text { Health Insurance } \\
\text { Adequacy, Black } \\
\text { (\%) No (\%) OR }(95 \% \mathrm{CI})\end{array}$} \\
\hline & OR $(95 \% \mathrm{CI})$ & Yes $(\%)$ No $(\%)$ & OR $(95 \% \mathrm{CI})$ & \\
\hline
\end{tabular}

Age

\begin{tabular}{|c|c|c|c|c|c|c|c|c|c|}
\hline $\begin{array}{l}\text { Between } 0-5 \\
6 \text {-11 years } \\
12-17 \text { years }\end{array}$ & $\begin{array}{l}81.5 \\
76.1 \\
74.1\end{array}$ & $\begin{array}{l}18.4 \\
23.9 \\
25.9\end{array}$ & $\begin{array}{c}1.00 \\
0.74(0.66,0.82) \\
0.69(0.61,0.76)\end{array}$ & $\begin{array}{l}82.0 \\
79.1 \\
74.1\end{array}$ & $\begin{array}{l}18.0 \\
20.2 \\
25.9\end{array}$ & $\begin{array}{c}1.00 \\
0.77(0.61,0.99) \\
0.69(0.54,0.91)\end{array}$ & $\begin{array}{l}79.8 \\
74.4 \\
71.9\end{array}$ & $\begin{array}{l}20.2 \\
25.6 \\
28.1\end{array}$ & $\begin{array}{c}1.00 \\
0.82(0.63,1.06) \\
0.65(0.51,0.84)\end{array}$ \\
\hline $\begin{array}{l}\text { Gender } \\
\text { Male } \\
\text { Female }\end{array}$ & $\begin{array}{l}77.0 \\
77.1\end{array}$ & $\begin{array}{l}23.0 \\
22.9\end{array}$ & $\begin{array}{c}1.00 \\
1.02(0.94,1.11)\end{array}$ & $\begin{array}{l}76.0 \\
80.2\end{array}$ & $\begin{array}{l}24.0 \\
19.8\end{array}$ & $\begin{array}{c}1.00 \\
1.14(0.93,1.40)\end{array}$ & $\begin{array}{l}74.3 \\
77.3\end{array}$ & $\begin{array}{l}25.7 \\
22.7\end{array}$ & $\begin{array}{c}1.00 \\
1.36(1.12,1.67)\end{array}$ \\
\hline $\begin{array}{l}\text { Type of health insurance } \\
\text { Public insurance } \\
\text { Private insurance }\end{array}$ & $\begin{array}{l}85.8 \\
74.1\end{array}$ & $\begin{array}{l}14.2 \\
25.8\end{array}$ & $\begin{array}{c}2.66(2.27,3.12) \\
1.00\end{array}$ & $\begin{array}{l}82.2 \\
72.1\end{array}$ & $\begin{array}{l}17.1 \\
27.9\end{array}$ & $\begin{array}{c}1.92(1.44,2.55) \\
1.00\end{array}$ & $\begin{array}{l}79.7 \\
68.8\end{array}$ & $\begin{array}{l}20.3 \\
31.2\end{array}$ & $\begin{array}{c}2.10(1.62,2.73) \\
1.00\end{array}$ \\
\hline $\begin{array}{l}\text { Poverty Level } \\
\text { 0-199\% FPL } \\
\text { 200-299\% FPL } \\
\text { 300-399\% FPL } \\
\text { 400\% FPL and above }\end{array}$ & $\begin{array}{l}79.8 \\
73.1 \\
72.9 \\
78.6\end{array}$ & $\begin{array}{l}20.2 \\
26.9 \\
27.1 \\
21.4\end{array}$ & $\begin{array}{c}0.68(0.59,0.79) \\
0.64(0.57,0.73) \\
0.72(0.64,0.81) \\
1.00\end{array}$ & $\begin{array}{l}78.8 \\
76.6 \\
77.2 \\
77.3\end{array}$ & $\begin{array}{l}21.2 \\
23.4 \\
22.8 \\
22.8\end{array}$ & $\begin{array}{c}0.93(0.66,1.31) \\
1.10(0.76,1.58) \\
0.86(0.55,1.34) \\
1.00\end{array}$ & $\begin{array}{l}77.4 \\
75.7 \\
67.8 \\
71.8\end{array}$ & $\begin{array}{l}22.6 \\
24.3 \\
32.2 \\
28.2\end{array}$ & $\begin{array}{c}0.58(0.42,0.81) \\
0.71(0.52,0.98) \\
0.83(0.57,1.20) \\
1.00\end{array}$ \\
\hline $\begin{array}{l}\text { Family structure } \\
\text { Two parents - biological } \\
\text { Two parents - step family } \\
\text { Single mother - no father } \\
\text { Other family type }\end{array}$ & $\begin{array}{l}76.5 \\
78.1 \\
77.7 \\
80.0\end{array}$ & $\begin{array}{l}23.5 \\
21.9 \\
22.2 \\
19.9\end{array}$ & $\begin{array}{c}1.00 \\
1.12(0.95,1.32) \\
1.00(0.87,1.15) \\
0.88(0.29,2.70)\end{array}$ & $\begin{array}{l}74.2 \\
78.2 \\
81.6 \\
77.7\end{array}$ & $\begin{array}{l}25.8 \\
21.8 \\
18.4 \\
22.3\end{array}$ & $\begin{array}{c}1.00 \\
1.17(0.80,1.72) \\
1.02(0.79,1.32) \\
39.8(4.11,384.7)\end{array}$ & $\begin{array}{l}73.4 \\
77.4 \\
76.9 \\
23.0\end{array}$ & $\begin{array}{l}24.6 \\
22.6 \\
23.0 \\
25.1\end{array}$ & $\begin{array}{c}1.00 \\
1.29(0.89,1.86) \\
1.43(1.13,1.81) \\
1.21(0.43,3.42)\end{array}$ \\
\hline $\begin{array}{l}\text { Maternal health status } \\
\text { (mental and physical) } \\
\text { BOTH Good/Excellent } \\
\text { BOTH Not Good/Excellent }\end{array}$ & $\begin{array}{l}78.9 \\
73.2\end{array}$ & $\begin{array}{l}21.1 \\
26.8\end{array}$ & $\begin{array}{c}1.00 \\
0.68(0.62,0.74)\end{array}$ & $\begin{array}{l}79.0 \\
77.3\end{array}$ & $\begin{array}{l}20.9 \\
22.8\end{array}$ & $\begin{array}{c}1.00 \\
0.73(0.58,0.91)\end{array}$ & $\begin{array}{l}77.9 \\
74.2\end{array}$ & $\begin{array}{l}22.1 \\
25.8\end{array}$ & $\begin{array}{c}1.00 \\
0.85(0.69,1.06)\end{array}$ \\
\hline
\end{tabular}


Table 4. Distribution of children's sociodemographic/family characteristics by health insurance consistency in 2011/12 NSCH.

\begin{tabular}{|c|c|c|c|}
\hline & Consistent & $\begin{array}{l}\text { ed Count (We } \\
\text { Inconsistent }\end{array}$ & P-value \\
\hline $\begin{array}{l}\text { Age } \\
0-5 \text { years old } \\
6-11 \text { years old } \\
12-17 \text { years old }\end{array}$ & $\begin{array}{l}23,290(93.4) \\
24,271(93.6) \\
27,948(94.5)\end{array}$ & $\begin{array}{l}1,259(6.6) \\
1,181(6.4) \\
1,101(5.5)\end{array}$ & 0.0802 \\
\hline $\begin{array}{l}\text { Gender } \\
\text { Male } \\
\text { Female } \\
\end{array}$ & $\begin{array}{l}38,963(93.9) \\
36,476(93.8)\end{array}$ & $\begin{array}{l}1,834(6.1) \\
1,703(6.2)\end{array}$ & 0.8289 \\
\hline $\begin{array}{l}\text { Race/Ethnicity } \\
\text { White } \\
\text { Hispanic } \\
\text { Black }\end{array}$ & $\begin{array}{l}56,970(95.5) \\
10,592(90.9) \\
7,947(92.1)\end{array}$ & $\begin{array}{l}2,172(4.5) \\
876(9.1) \\
493(7.9)\end{array}$ & 0.0000 \\
\hline $\begin{array}{l}\text { Type of Healthcare insurance } \\
\text { Public insurance } \\
\text { Private Insurance }\end{array}$ & $\begin{array}{l}20,894(88.7) \\
54,016(97.2) \\
\end{array}$ & $\begin{array}{c}2,204(11.3) \\
1,294(2.8)\end{array}$ & 0.0000 \\
\hline $\begin{array}{l}\text { Poverty Level } \\
\text { 0-199\% FPL } \\
\text { 200-299\% FPL } \\
300-399 \% \text { FPL } \\
\text { 400\% FPL and above }\end{array}$ & $\begin{array}{l}22,879(90.6) \\
12,068(93.0) \\
11,334(96.0) \\
29,228(98.3)\end{array}$ & $\begin{array}{l}2,125(9.4) \\
659(7.0) \\
309(4.0) \\
448(1.7)\end{array}$ & 0.0000 \\
\hline $\begin{array}{l}\text { Family Structure } \\
\text { Two parents - biological } \\
\text { Two parents - step family } \\
\text { Single mother - no father } \\
\text { Other family type }\end{array}$ & $\begin{array}{c}53,828(94.9) \\
5,075(92.0) \\
11,449(91.5) \\
4,801(93.3)\end{array}$ & $\begin{array}{l}1,939(5.1) \\
336(8.0) \\
899(8.5) \\
341(6.7)\end{array}$ & 0.0000 \\
\hline $\begin{array}{l}\text { Maternal health status (mental and physical) } \\
\text { BOTH Good/Excellent } \\
\text { BOTH Not Good/Excellent }\end{array}$ & $\begin{array}{l}44,509(95.4) \\
26,177(91.8)\end{array}$ & $\begin{array}{l}1,475(4.6) \\
1,723(8.2)\end{array}$ & 0.0000 \\
\hline
\end{tabular}

Table 5. Weighted analysis of health insurance consistency and Odds Ratios (OR) (95\% Confidence Intervals) by race/ethnicity among children in 2011-2012 NSCH.

\begin{tabular}{|c|c|c|c|c|c|c|c|c|c|}
\hline \multirow{2}{*}{. } & \multicolumn{3}{|c|}{$\begin{array}{l}\text { Health Insurance } \\
\text { Consistency, White }\end{array}$} & \multicolumn{3}{|c|}{$\begin{array}{l}\text { Health Insurance } \\
\text { Consistency, Hispanic }\end{array}$} & \multicolumn{3}{|c|}{$\begin{array}{l}\text { Health Insurance } \\
\text { Consistency, Black }\end{array}$} \\
\hline & Yes $(\%)$ & No $(\%)$ & OR $(95 \% \mathrm{CI})$ & Yes (\%) & No $(\%)$ & OR $(95 \% \mathrm{CI})$ & Yes $(\%)$ & No $(\%)$ & OR (95\% CI) \\
\hline \multicolumn{10}{|l|}{ Age } \\
\hline $0-5$ years old & 91.5 & 8.4 & 1.00 & 87.8 & 12.2 & 1.00 & 84.4 & 15.5 & 1.00 \\
\hline 6 -11 years old & 91.5 & 8.4 & $0.90(0.70,1.14)$ & 86.9 & 13.2 & $0.96(0.66,1.38)$ & 81.7 & 18.6 & $1.15(0.71,1.88)$ \\
\hline $12-17$ years old & 92.2 & 7.7 & $1.03(0.80,1.32)$ & 87.9 & 12.1 & $0.95(0.62,1.46)$ & 79.6 & 20.4 & $0.89(0.57,1.39)$ \\
\hline \multicolumn{10}{|l|}{ Gender } \\
\hline Male & 91.8 & 8.2 & 1.00 & 88.5 & 11.4 & 1.00 & 80.6 & 19.6 & 1.00 \\
\hline Female & 91.9 & 8.0 & $0.98(0.81,1.20)$ & 86.5 & 13.4 & $1.10(0.79,1.52)$ & 83.8 & 16.2 & $0.76(0.54,1.07)$ \\
\hline \multicolumn{10}{|l|}{ Type of health insurance } \\
\hline Public insurance & 89.9 & 10.5 & $0.36(0.27,0.48)$ & 89.6 & 10.4 & $0.29(0.18,0.46)$ & 87.6 & 12.4 & $0.32(0.19,0.53)$ \\
\hline Private insurance & 97.5 & 2.48 & 1.00 & 95.9 & 4.1 & 1.00 & 96.4 & 3.63 & 1.00 \\
\hline \multicolumn{10}{|l|}{ Poverty Level } \\
\hline $0-199 \%$ FPL & 84.8 & 15.2 & $0.32(0.23,0.45)$ & 85.5 & 14.5 & $0.48(0.25,0.93)$ & 78.8 & 21.2 & $0.79(0.39,1.587)$ \\
\hline 200-299\% FPL & 89.9 & 10.0 & $0.31(0.22,0.43)$ & 88.0 & 11.9 & $0.26(0.13,0.56)$ & 80.6 & 19.4 & $0.64(0.33,1.25)$ \\
\hline 300-399 FPL & 94.3 & 5.6 & $0.45(0.31,0.65)$ & 91.1 & 8.9 & $0.56(0.22,1.46)$ & 89.7 & 10.3 & $0.50(0.17,1.49)$ \\
\hline 400\% FPL and above & 97.4 & 2.6 & 1.00 & 93.1 & 6.8 & 1.00 & 96.3 & 3.7 & 1.00 \\
\hline \multicolumn{10}{|l|}{ Family Structure } \\
\hline Two parents - biological & 92.9 & 7.0 & 1.00 & 87.9 & 12.1 & 1.00 & 82.4 & 17.5 & 1.00 \\
\hline Two parents - step family & 89.3 & 10.6 & $0.87(0.61,1.23)$ & 85.9 & 14.0 & $0.76(0.43,1.33)$ & 78.0 & 21.9 & $0.96(0.52,1.78)$ \\
\hline Single mother - no father & 87.8 & 12.2 & $0.91(0.70,1.18)$ & 86.7 & 13.3 & $1.19(0.83,1.72)$ & 83.3 & 16.6 & $1.09(0.69,1.72)$ \\
\hline Other family type & 90.3 & 8.2 & $1.67(0.47,5.90)$ & 90.3 & 9.6 & $1.07(0.12,9.41)$ & 77.5 & 17.9 & $5.52(0.59,50.78)$ \\
\hline \multicolumn{10}{|c|}{ Maternal health status (mental and physical) } \\
\hline BOTH Good/Excellent & 93.4 & 10.8 & 1.00 & 88.8 & 14.5 & 1.00 & 86.4 & 20.1 & 1.00 \\
\hline BOTH Not Good/Excellent & 89.1 & 6.5 & $0.84(0.69,1.03)$ & 85.4 & 11.2 & $0.64(0.45,0.91)$ & 79.1 & 13.5 & $1.04(0.73,1.48)$ \\
\hline
\end{tabular}


healthcare services.

Furthermore, the findings of this study show that both black and white children living in low-income families may face challenges in obtaining adequate health insurance coverage. At a national level, over $65 \%$ of black children and around $30 \%$ of white children live in low-income households ${ }^{24}$ with black children experiencing higher levels of financial burdens, ${ }^{18}$ which could contribute to both underinsurance and inconsistent coverage. ${ }^{9}$ Although previous studies consistently show that poverty negatively affects adequate health care coverage in black children, ${ }^{18,25}$ research also needs to address mechanisms by which poverty affects access to health care and services among white children residing in low-income households.

In the present study, for Hispanic and white children poor maternal health was associated with a decreased likelihood of adequate health insurance. Generally, poor parental health and especially that of the mother has been linked to poor children's health outcomes. ${ }^{26}$ It is possible that the common link between these events is underinsurance and lack of insurance consistency.

Regardless of race/ethnicity, we found that children with public healthcare insurance were more likely to have adequate insurance than their privately insured counterparts. This finding is consistent with previous research that has documented an increase in adequate insurance levels among publicly insured children. ${ }^{27,28}$ This could be due to Medicaid and CHIP Reauthorization Act of 2009 (CHIPRA). However, we found that children with public insurance were less likely to have consistent health insurance coverage compared to children with private health insurance. This highlights the fact that children on public health insurance may be more vulnerable to inconsistent health insurance coverage and hence intermittent access to healthcare. A recent cross-sectional study analyzed the reasons for lacking health insurance among minority children who were eligible for public insurance (Medicaid/CHIP) and found that the most common reason for insurance inconsistency was expiration of benefits and never reapplied. ${ }^{18}$ Our findings on publicly insured children indicate the urgent need for improved focus on children's continuation of healthcare services.

Although we did not find a significant association between race/ethnicity and insurance adequacy in bivariate analyses, Hispanic and Black children were more likely to lack consistent insurance coverage. These findings are in agreement with previous research studies that show minority children experiencing higher levels of inconsistent insurance coverage. ${ }^{9,18}$ An explanation of these findings can be drawn from the sociodemographic and family related characteristics of these children. We found that both black and Hispanic children had a high likelihood of living in poverty, having public insurance and residing in households with poor parental health, all of which can place a child at risk for inconsistent insurance coverage. In stratified analyses by race/ethnicity, we found that Hispanic and white children living in lower-income households were more likely to lack consistent insurance coverage. Studies have shown that although children of lower-income levels may be able to obtain health insurance via different programs (e.g., Children's Health Insurance Program-CHIP), they are significantly more likely to be inconsistently insured. ${ }^{29}$ In previous studies, lack of consistent insurance among minority children has been associated with limited English proficiency, complex enrollment processes and families not being aware of their eligibility for CHIP programs. ${ }^{30}$ Further, a recent study indicated that expanding CHIP public insurance to minority children would increase coverage by $24.5 \%$, improve access to care, and reduce disparities substantially. ${ }^{31}$ This could also be extended to white children residing in low-income households.

Several study limitations are present in this study. The NSCH is a cross-sectional survey and consequently cannot measure racial/ethnic differences in insurance adequacy and consistency lon- gitudinally. The definitions of insurance adequacy and consistency capture access to healthcare partly as insurance coverage is multidimensional. Further, several measures used in this study were selfreported and were associated with telephone surveys which are subject to biases. For example, parental or guardian responses to some of the survey questions might be biased depending on their knowledge and perceptions of whether their children's health insurance covers needed services. The strengths of this study include the use of a large nationally representative sample and measures of both insurance adequacy and consistency.

\section{Conclusions}

In this nationally representative sample of children, differences and similarities were observed in contributing factors to insurance adequacy and consistency among children of different racial/ethnic backgrounds. Low-income and poor maternal health adversely affected insurance consistency and adequacy for both minority and white children. Considering the lack of health insurance adequacy among adolescents, policy makers should consider extending health insurance coverage for this older group of children. Research studies on inequalities of healthcare coverage should also monitor underserved white populations of children as their insurance coverage is affected by similar factors as those for minority children. Elimination of inequalities may require targeted interventions that include cross-cultural education of healthcare providers, policy changes to grant low-income children with appropriate and reliable health insurance, research that may shed some light on the underlying mechanisms of underinsurance/inconsistency of insurance in minority populations, and an ongoing monitoring of disparities by health plans (e.g., CHIP, Medicaid, private insurance).

Correspondence: Panagiota Kitsantas, Department of Health Administration and Policy, George Mason University, 4400 University Drive, MS 1J3, Fairfax, VA 22030-4444, USA.

Tel.: + 1.703.993.9164.

E-mail: pkitsant@gmu.edu

Key words: Health insurance adequacy; health insurance consistency; race/ethnicity; children; sociodemographic and family factors.

Contributions: TGS coordinated the study by providing timely information and feedback, keeping the research progress within the time line, writing and editing the paper, and formatting the manuscript in line with the journal requirements; EE contributed by coordinating the selection of the variables, coding, and entering them into Stata software for analysis; FA contributed by conducting extensive literature review, writing the paper, and editing the overall paper; MA contributed by coordinating the selection of the variables, coding, and data analysis; PK is the senior member of the team who had a role of overseeing the study by providing a continuous guidance and direction to team members.

Conflict of interest: the authors declare no potential conflict of interest.

Funding: none.

Received for publication: 23 0ctober 2017.

Revision received: 14 April 2018.

Accepted for publication: 14 April 2018.

(C) Copyright T.G. Soylu et al., 2018

Licensee PAGEPress, Italy

Journal of Public Health Research 2018;7:1280

doi:10.4081/jphr.2018.1280

This work is licensed under a Creative Commons Attribution NonCommercial 4.0 License (CC BY-NC 4.0). 
Pediatrics 2009;123:191-6.

\section{References}

1. CDC. National Health Interview Survey Early Release, 2015. Available from: https://www.cdc.gov/nchs/data/nhis/earlyrelease/earlyrelease201605.pdf

2. Jeffrey AE, Newacheck PW. Role of insurance for children with special health care needs: a synthesis of the evidence. Pediatrics 2006;118:e1027-38.

3. Szilagyi PG, Schuster MA, Cheng TL. The scientific evidence for child health insurance. Acad Pediatr 2009;9:4-6.

4. Federico SG, Steiner JF, Beaty B, et al. Disruptions in insurance coverage: patterns and relationship to health care access, unmet need, and utilization before enrollment in the state children's health insurance program. Pediatrics 2007;120:e100916.

5. Cassedy A, Fairbrother G, Newacheck PW. The impact of insurance instability on children's access, utilization, and satisfaction with health care. Ambul Pediatr 2008;8:321-8.

6. Halterman JS, Montes G, Shone LP, Szilagyi PG. The impact of health insurance gaps on access to care among children with asthma in the United States. Ambul Pediatr 2008;8:43-9.

7. Flores G, Tomany-Korman SC. Racial and ethnic disparities in medical and dental health, access to care, and use of services in US children. Pediatrics 2008;121:e286-98.

8. US Census Bureau, Current Population Survey. Health insurance coverage status and type of coverage by selected characteristics for children under 18. 2014.). Available from https://www.census.gov/hhes/www/cpstables/032015/health/t oc.htm.

9. Kogan MD, Newacheck PW, Blumberg SJ, et al. Underinsurance among cildren in the United States. N Engl J Med 2010;63:841-51.

10. Flores G, Lin H. Trends in racial/ethnic disparities in medical and oral health, access to care, and use of services in US children: has anything changed over the years? Int J Equity Health 2013;12:10.

11. US Government. ACA Implementation. Available from: whitehouse.gov.

12. Adepoju OE, Preston MA, Gonzales G. Health Care Disparities in the Post-Affordable Care Act Era. Am J Public Health 2015;105;S665-7.

13. American College of Physicians. Racial and Ethnic Disparities in Health Care, Updated 2010. Available from: www.acponline.org/acp_policy/policies/racial_ethnic_disparities_2010.pdf.

14. CDC. National Survey of Children's Health. 2011. Available from: https://www.cdc.gov/nchs/data/ slaits/2011NSCH Questionnaire.pdf).

15. National Survey of Children's Health (NSCH). Guide to topics \& questions asked. 2011/12. Available from: http://www.c hildhealthdata.org.

16. American Academy of Pediatrics. Underinsurance of Adolescents: recommendations for improved coverage of preventive, reproductive, and behavioral health care services.
17. Bethell CD, Kogan MD, Strickland BB, et al. A national and state profile of leading health problems and health care quality for US children: key insurance disparities and across-state variations. Acad Pediatr 2011;11:S22-33.

18. Flores G, Lin H, Walker C, et al. A cross-sectional study of parental awareness of and reasons for lack of health insurance among minority children, and the impact on health, access to care, and unmet needs. Int J Equity Health 2016;15:s12939.

19. King C. Disparities in access to preventive health care services among insured children in a cross-sectional study. Medicine 2016;95:e4262.

20. Abdus S, Hudson J, Hill SC, Selden TM. Children's health insurance program premiums adversely affect enrollment, especially among lower-income children. Health Affairs 2014;1353-1360.

21. Saloner B, Koyawala N, Kenney GM. Coverage for low-income immigrant children increased 24.5 percent in states that expanded CHIPRA eligibility. Health Affairs 2014;33:832-9.

22. Angier H, DeVoe JE, Tillotson C, et al. Trends in Health Insurance Status of US Children and their Parents, 1998-2008. Maternal Child Health J 2012;17:1550-8.

23. STATA: StataCorp. 2015. Stata Statistical Software: Release 14. College Station, TX: StataCorp LP.).

24. Jiang Y, Ekono M, Skinner C. Basic facts about low-income children under 18 years, 2014. New York: National Center for Children in Poverty, 2016; Mailman School of Public Health, Columbia University.

25. Smith JC, Medalia C. US Census Bureau. Health insurance coverage in the United States: Washington, DC: U.S. Government Printing Office; 2014. Available from: https://www.census.gov/content/dam/Census/library/publications/2015/demo/p60-253.pdf.

26. Kitsantas P, Kornides M, Cantiello J, Wu H. Chronic physical health conditions among children of different racial/ethnic backgrounds. Public Health 2013;127:546-53.

27. Ghandour RM, Comeau M, Tobias C, et al. Assuring adequate health insurance for children with special health care needs: progress from 2001 to 2009-2010. Acad Pediatr 2015;15:45160.

28. Howell EM, Kenney GM. The impact of the medicaid/CHIP expansions on children. Med Care Res Rev 2012;69:372-96.

29. Flores G, Lin H. Trends in racial/ethnic disparities in medical and oral health, access to care, and use of services in US children: has anything changed over the years? Int J Equity Health 2013; $12: 10$.

30. American College of Physicians. Racial and Ethnic Disparities in Health Care, Updated 2010. Available from: www.acponline.org/acp_policy/policies/racial_ethnic_disparities_2010.pdf.

31. Saloner B, Koyawala N, Kenney GM. Coverage for low-income immigrant children increased 24.5 percent in states that expanded CHIPRA eligibility. Health Affairs 2014;33:832-9. 